\title{
Smart Health and Wellbeing
}

\author{
CHRISTOPHER C. YANG, Drexel University \\ GONDY LEROY, University of Arizona \\ SOPHIA ANANIADOU, University of Manchester
}

Healthcare informatics has drawn substantial attention in recent years. Current work on healthcare informatics is highly interdisciplinary involving methodologies from computing, engineering, information science, behavior science, management science, social science, as well as many different areas in medicine and public health. Three major tracks, (i) systems, (ii) analytics, and (iii) human factors, can be identified. The systems track focuses on healthcare system architecture, framework, design, engineering, and application; the analytics track emphasizes data/information processing, retrieval, mining, analytics, as well as knowledge discovery; the human factors track targets the understanding of users or context, interface design, and user studies of healthcare applications. In this article, we discuss some of the latest development and introduce several articles selected for this special issue. We envision that the development of computing-oriented healthcare informatics research will continue to grow rapidly. The integration of different disciplines to advance the healthcare and wellbeing of our society will also be accelerated.

Categories and Subject Descriptors: H.1.2 [Models and Principles]: User/Machine Systems-Human factors; H.2.0 [Database Management]: General-Security, integrity, and protection; H.2.8 [Database Management]: Database Applications-Data mining; H.3.1 [Information Storage and Retrieval]: Content Analysis and Indexing-Linguistic processing; H.3.3 [Information Storage and Retrieval]: Information Search and Retrieval; H.5.1 [Information Interfaces and Presentation]: Multimedia Information Systems-Artificial, augmented, and virtual realities; evaluation/methodology; H.5.2 [Information Interfaces and Presentation]: User Interfaces—Graphical user interfaces; H.5.4 [Information Interfaces and Presentation]: Hypertext/Hypermedia; I.2.7 [Artificial Intelligence]: Natural Language Processing-Text analysis; J.3 [Life and Medical Sciences]: Health

General Terms: Algorithms, Design, Experimentation, Human Factors, Security

ACM Reference Format:

Yang, C. C., Leroy, G., and Ananiadou, S. 2013. Smart health and wellbeing. ACM Trans. Manage. Inf. Syst. 4, 4, Article 15 (December 2013), 8 pages.

DOI : http://dx.doi.org/10.1145/2555810.2555811

\section{INTRODUCTION}

Health and wellbeing are very dear to people's hearts, something that is obvious from the many greetings used in almost all cultures containing wishes for good health. As such, it is no surprise that healthcare plays an important role in our societies. Improving the health and wellbeing of people is a main goal set forth by both government and private organizations. Over the decades, healthcare, medicine, surgery, and most other related healthcare activities have increased and improved.

While our ultimate goal is broadly the same, increasing health and wellbeing, there are many paths to accomplishing this and many different disciplines that contribute to it. In this special issue, we aim to bring a few examples of new approaches to further

Author's address: C. C. Yang, Drexel University; email: chris.yang@drexel.edu.

Permission to make digital or hard copies of part or all of this work for personal or classroom use is granted without fee provided that copies are not made or distributed for profit or commercial advantage and that copies show this notice on the first page or initial screen of a display along with the full citation. Copyrights for components of this work owned by others than ACM must be honored. Abstracting with credit is permitted. To copy otherwise, to republish, to post on servers, to redistribute to lists, or to use any component of this work in other works requires prior specific permission and/or a fee. Permissions may be requested from Publications Dept., ACM, Inc., 2 Penn Plaza, Suite 701, New York, NY 10121-0701 USA, fax +1 (212) 869-0481, or permissions@acm.org.

(c) 2013 ACM 2158-656X/2013/12-ART15 $\$ 15.00$

DOI : http://dx.doi.org/10.1145/2555810.2555811 
advancing our health and wellbeing. With our call for papers, we charged authors to bring work that presented novel approaches, combined with a quality evaluation, to allow readers to understand and evaluate the work and hopefully also to build on the discoveries and use them to further the field.

\section{HEALTHCARE INFORMATICS EVOLUTION}

With increasing processing power, storage capacity, advanced algorithms and a willingness to use and integrate computer-based solutions in healthcare, the influence of information technology on health and wellbeing has increased considerably. While early on, its impact and application may have been restricted to data analysis or to supporting highly specialized interventions, information technology now touches all aspects of healthcare and wellbeing.

All parties involved in the healthcare of an individual use information technology. It is used by clinicians and hospitals for administrative purposes, for example, with the increased use and focus on electronic health records (EHR), but naturally also for diagnosis, treatment, and therapy, particularly with the heavy use of imaging and video in medicine. Insurance companies use information management systems for data and information processing, especially billing, but increasingly also to transfer and share information such as test results, to e-prescribe medications, and to send public health messages to their customers. For researchers, there have been revolutions that have advanced their work and enabled entirely new medical possibilities, such as personalized medicine, in silico experimentation, and increased and improved access to data for research. Not only are more parties affected, the scope of information technology systems has also been broadened. Early on, the focus was on specialized applications, such as telemedicine to support remote diagnosis or algorithms trained to distinguish between malignant and benign masses. More recently, these are included as components in much more advanced decision-support systems. Effects on both the short-term and long-term are being tracked.

While the preceding lists are examples of common and historical use of information technology in healthcare, there have been two especially noteworthy changes that have evolved during the last decade. The first is the influence of information systems in the wider community that is concerned with one's healthcare. It has extended beyond the medical care providers. Patients and their family members accept and expect the use of information technology. For example, many prefer to schedule and reschedule appointments online, to communicate with their caregivers by email, and to use storage devices to personally store their records and diagnostic tests. Patients also use computer-based support tools to manage or improve their conditions, for example, in chronic diseases such as diabetes. And increasingly, they share information, advice, and support via online communities with medical professionals and even more often with fellow patients, such as MedHelp.org and PatientsLikeMe.com, or with family members, for instance, Caringbridge.org.

A second striking change is the acceptability of technology beyond medical interventions, including their integration in preventive health programs and their use in movements aimed at keeping people healthy and supporting the wellbeing of users of all ages and interests. Providers and developers are collaborating to develop systems to enhance the quality of life and support independence. For example, for vulnerable groups, such as children with severe autism, these apps and online learning systems in development that will teach them to communicate, practice social skills and integration into the community, and grow up to be independent and healthy. Others are working on providing tools to increase reader comprehension of medical information, both text and numeric, especially for vulnerable groups such as those with low heal th literacy or high stress. Furthermore, the increased interest is not limited to initiatives 
by healthcare providers; new technology is often popularized by laymen. For example, trackers of all kinds are common and popular. Many websites or apps provide calorie trackers, glucose, fertility, and fitness trackers for a nominal fee or for free. Information technology for increasing wellbeing and health has become a lifestyle and necessary accessory for many.

\section{HEALTHCARE INFORMATICS RESEARCH FRAMEWORK}

Healthcare informatics research is highly interdisciplinary. It involves research methodologies from computing, engineering, information science, behavior science, management science, social science, and different areas of medicine and public health. In general, we can identify three major tracks of research work in healthcare informatics: (i) systems, (ii) analytics, and (iii) human factors. Work on systems focuses on healthcare system architecture, framework, design, engineering, and application; the analytics track encompasses data/information processing, retrieval, mining, and analytics, as well as knowledge discovery; and the human factors track focuses on understanding users or context, interface design, and user studies of healthcare applications. In this section, we summarize a few major and recent research topics in healthcare informatics.

\subsection{Systems}

When information systems are put into place, several aspects beyond the simple working of individual algorithms become important. There are many systems that combine different data sources or apply algorithms that do not directly touch the lives of, or interact with, patients. Other systems are meant to augment lives and lifestyles directly. In both cases, the reliability of the underlying technology is of the utmost importance. Medicine is considered a safety-first field and new approaches need to be measured against existing approaches accepted as safe and show demonstrated improvements over the existing approaches.

An example of the first type of system can be found in medical health record systems. The information sharing and capturing that these systems provide can reduce costs and bring new opportunities when datasets are integrated, but they also bring added concerns about privacy and security. Information sharing is beneficial in improving healthcare quality and encouraged in the Health Information Technology for Economic and Clinical Health Act (HITECH). In addition, the advance of sensor and communication technologies allows the capturing of ubiquitous and personalized healthcare data for personalization or information aggregation. However, sharing electronic health records among healthcare providers or with the research community raises concerns. An example of a response to these concerns was the creation of the Health Insurance Portability and Accountability ACT (HIPAA), which specifies strict requirements on appropriate access control for identifiable healthcare information. Access control modeling [Alshehri and Raj 2013; Khan and McKilop 2013] has been developed to assure the security and privacy of patient data while the patient data is collected, used, and shared across multiple healthcare data custodians. Others attempt to discover new knowledge or make recommendations through accessing multiple healthcare data sources, while, at the same time, protecting the privacy of patients [Hoens et al. 2013; Park and Ghosh 2013]. Techniques that focus on protecting the privacy of video and image data for home-monitoring systems and medical-imaging systems have also been investigated [Mehta et al. 2013].

Many systems in which the patient and technology interact are being put into place. Excellent examples are found in assisted living and personalized care. The aging population is increasing in most developed and developing countries due to advances in medicine. At the same time, the demand for assisted living for the elderly is also 
increasing, resulting in a shortage of appropriately trained healthcare workers and an increase in healthcare costs. Likewise, advances in medicine prolong the life expectancy of many patients with chronic illnesses, for example, with HIV. The demand for personalized care for chronic patients is also growing substantially. A variety of technology-enabled approaches are being developed to support personalized care and improve both assisted and independent living. Telemedicine and clinical homemonitoring systems are being investigated to detect potential risks. For example, fall detection, using radar signals and video monitoring, has been studied widely [Dai et al. 2013]. Recent research has also investigated how to utilize robots, sensors, the Internet, and mobile technologies for personalized care [Juarez et al. 2013; Rashidi and Cook 2013]. Most of these projects aim to assist the elderly and chronic patients so that they can take care of themselves more independently and increase their quality of life, while reducing healthcare costs at the same time.

\subsection{Analytics}

With a renewed interest in data processing and the associated popularity of data science and big data, it is no surprise that this trend also exists in healthcare. More and larger datasets are being created (e.g., www.healthdata.gov), and several open source products facilitate access and analysis of this data (e.g., Weka at http://www.cs.waikato.ac.nz/ml/weka/, RapidMiner at http://rapidminer.com/, among many others that can be found at http://www.kdnuggets.com/). Especially in biomedicine, this movement is accepted, encouraged, and even expected. For example, several journals request submission of data or experimental setups with manuscript submissions. In contrast to the biomedical data, the access to patient data through electronic health records is making slower progress. However, with their increasing usefulness becoming apparent, they will ultimately also be utilized to search for costeffective and efficient interventions, and they may also help bring transparency to the field, for instance, with medical errors, so that lessons can be learned from them. In the following, we briefly touch on a few remarkable and interesting developments.

Data from structured sources has become abundant, making prediction algorithms possible. For example, healthcare predictive modeling and risk assessment are widely used in healthcare informatics for assessing risk factors associated with a disease, predicting disease progression, and even identifying patients at risk of hospital (re)admission [Ho et al. 2013; Lemke 2013; Yu et al. 2013]. This is particularly important for those diseases whose cause and nature are still unknown. For these, example risk factors assessed are genetic, environmental, and dietary factors as well as health behavior and comorbidity. These are used to develop predictive models often using probabilistic analysis, machine learning algorithms, and scoring systems. For example, hospital readmissions contribute a substantial amount to the high healthcare cost, and the Hospital Readmission Reduction Program (HRRP) was introduced in 2012 to penalize hospitals that have excessive readmissions. In recent years, many readmission prediction models have been developed, which are helpful in managing the hospital discharge process and outpatient care transition. Some focus on the role of technology in healthcare and how it can help reduce readmissions [Bardha et al. 2011].

Another excellent example of new developments in data analytics is drug management and development. Drug management is important because post-marketing surveillance is a main source of data for further evaluation of new drugs being developed and used. The process complements the limited clinical and laboratory tests that were done during the pre-marketing review. Not surprisingly, the analysis of pharmaceutical products relies on many different data sources, including electronic health records, laboratory/clinical tests, spontaneous reporting systems, pharmaceutical/life 
science databases, and online health communities [Yang et al. 2012]. For example, analytics techniques are used to extract drug-disease relationship [Khare et al. 2013], adverse drug reaction [Yang et al. 2012], drug-drug interactions [Yang and Yang 2013], and drug repurposing activities.

In addition to structured data sources, unstructured sources, that is texts, are increasingly used as the basis for text mining and analytics. There exists an overwhelming and rapidly growing amount of information in unstructured textual form. For example, the PubMed database of biomedical literature, which currently contains about 23 million citations, is growing at the rate of approximately two pages per minute. There are also many free text fields within Electronic Health Records (EHRs), such as patient notes or discharge summaries, that require natural language processing and information extraction. The sheer volume of available text means that simple keyword-based searches, which lack expressive power, are not sufficient to allow relevant information to be located and extracted in a timely manner.

Text mining (TM) has the potential to alleviate the problems of information overload. Through the application of linguistic processing methods, TM aims to derive structured, semantic information from the content of documents so that they are no longer viewed as sets of unrelated words [Ananiadou and McNaught 2006]. Several approaches exist to extract predicates from terms, either based on generic grammar structures [Kwak et al. 2013] or focused on specific relationships of interest [Rindflesch and Fiszman 2003]. As a response to the maturing of the field, several initiatives have been started that aim to test and compare different approaches for standardized tasks. For example, the ongoing series of $\mathrm{i} 2 \mathrm{~b} 2^{1}$ and CLEF (Conference and Labs of the Evaluation Forum, http://www.clef2013.org/) natural language processing challenges are helping to encourage the development of various TM technologies for the healthcare domain.

Specific techniques like recognition of abbreviations or named entities (NEs) [Sasaki et al. 2008], such as genes, diseases, and drugs, can significantly improve search capabilities. NE recognition can also be used to facilitate the automatic clustering of documents (e.g. Korkontzelos et al. 2012), such that users can explore groups of documents with related themes. Building on NE detection, the extraction of relationships that exist between them is important and useful, for instance, gene-disease associations, drug-drug interactions, or relations between problems, tests, and treatments. The ability to search for cooccurrences of particular types of terms in documents can reveal potentially undiscovered associations, while the application of more sophisticated deep parsing technology [Miyao and Tsujii 2008], such as, SemRep [Rindflesch and Fiszman 2003] and event extraction techniques [Miwa et al. 2012], aim to detect more complex relationships between entities.

\subsection{Human Factors}

Human factors research strives to understand the human behavior when users, including health professionals and patients, interact with healthcare information systems. The goals are to reduce the use errors, as well as enhance the usability or effectiveness of healthcare applications. Use errors of healthcare devices or information systems may lead to moderate or great disability and even death. These use errors are often unnoticed errors that cannot be managed by clinicians but can be prevented by safer design [Thimbleby 2013]. Due to the latest developments in electronic health records, many researchers are looking into the usability of electronic health records systems from the patient perspective. The advances in mobile technologies in recent years also

$\overline{1_{\mathrm{https}} / / / \mathrm{www} . \mathrm{i} 2 \mathrm{~b} 2 . \mathrm{org} / \mathrm{NLP} /}$ 
draws attention to the need to study the effectiveness of mobile apps and social media in healthcare and how they may change the awareness of wellbeing and prevent diseases.

\section{RECENT ACTIVITIES}

In recent years, there has been a growing number of ACM and IEEE conferences and workshops related to healthcare informatics. These events focus on computing and engineering approaches in tackling healthcare problems and cover the topics mentioned in the last section. These conferences and workshops include but are not limited to the following.

- ACM SIGHIT International Health Informatics Symposium (IHI) 2010 and 2012

- ACM SIGKDD Workshop on Health Informatics (HI-KDD) 2012

- ACM CIKM International Workshop on Smart Health and Wellbeing (SHB) 2012

- ACM SIGKDD Workshop on Data Mining for Healthcare (DMH) 2013

— ACM SIGIR Workshop on Health Search and Discovery (HSD) 2013

- ACM Health Informatics Symposium in conjunction with ACM Conference on Bioinformatics, Computational Biology and Biomedical Informatics (BCB) 2013

— SDM Workshop on Data Mining for Medicine and Healthcare (DMMH) 2013

- IEEE ICDM Data Mining in Biomedical Informatics and Healthcare Workshop (DMBIH) 2013

— IEEE International Conference on Healthcare Informatics (ICHI) 2013

Other than these recent events, the Conference on Artificial Intelligence in Medicine (AIME) has been held for more than a decade. AIME is a European conference, founded by the European Society for Artificial Intelligence in Medicine, to foster fundamental and applied research in the application of artificial intelligence techniques to medical care and medical research. For decades, the American Medical Informatics Association (AMIA) has been hosting the AMIA Annual Symposium to bring together biomedical and health informatics researchers and practitioners; it focuses on the development and use of information to improve individual health, health care, public health, and to assist biomedical research across the continuum from molecules to populations.

\section{ARTICLES IN THE SPECIAL ISSUE}

There were three articles of this special issue published in an earlier issue, Volume 4, Issue 3. In "Distributed Privacy Preserving Decision Support System for Highly Imbalanced Clinical Data," George Mathew and Zoran Obradovic presented a distributed classification model that used the statistics of data from multiple hospitals and yet preserved the patient privacy. The system was able to deal with imbalanced data that was common in rare symptoms.

Masato Sakata, Zeynep Yücel, Kazuhiko Shinozawa, Norihiro Hagita, Michita Imai, Michiko Furutani, and Rumiko Matsuoka, in their article "An Inference Engine for Estimating Outside States of Clinical Test Items," utilized the multivariate kernel density estimate in a Bayesian framework to determine the relation between the states of a set of standard test items in common health check-ups and the future states of a set of costly medical tests. Their technique was able to enhance early detection of several diseases and improve healthcare quality.

In their article, "Accurate and Efficient Algorithms that Adapt to Privacy-Enhanced Video for Improved Assistive Monitoring," Alex Edgcomb and Frank Vahid presented two adaptive algorithms for improving assistive monitoring goal performance with privacy-enhanced video. The non-adaptive algorithms would degrade the performance of monitoring goals from raw video, but the experiment showed that the proposed adaptive algorithms made substantial improvements in accuracy. 
An additional five papers are included in this issue. In their paper, "Modeling Throughput of Emergency Departments via Time Series: An Expectation Maximization Algorithm," Zidong Wang, Julie Eatock, Sally McClean, Dongmei Lui, Xiaohui Liu, and Terry Young apply the expectation maximization algorithm to model the throughput of an emergency department through time series data from West London, in which the model parameters, the actual throughput, and the noise intensity were identified simultaneously.

In "Mining Deviations from Patient Care Pathways via Electronic Medical Record System Audits," He Zhang, Sanjay Mehotra, David Liebovitz, Carl Gunter, and Bradley Malin model the patient care pathway as a sequence of accesses defined over a graph, in which the elements in a sequence correspond to features associated with the access transactions of electronic medical record systems. They conducted empirical analysis on several months of data from a large academic medical center and found that a small portion of access constitutes outliers from typical care workflows. The result suggest that greater deviation from normal access patterns was made by non-clinical users.

The article by Rajiv Khosla and Mei-Tai Chiu, "Embodying Care in Matilda - An Affective Communication Robot for Emotional Wellbeing of Older People in Australian Residential Care Facilities," reports on the design and implementation of a service and companion robot for improving the emotional wellbeing of elderly patients. Positive outcomes were observed by engaging these patients.

In "I Can Help You Change! An Emphatic Virtual Agent Delivers Behavior Change Health Interventions," Christine Lisetti, Reza Amini, and Uqan Yasavur present the design and system architecture of a personalized on-demand virtual counseling system. The system adopt a multimodal embodied conversational agent to deliver behavior change intervention empathically. The user evaluation showed that the users had a more positive attitude toward the counseling system as well as several other dimensions of the users' acceptability such as intention to use, perceived enjoyment, perceived sociability, etc.

Finally, Rajesh Mirani and Anju Harpalani, authors of "Business Benefits or Incentive Maximization? Impacts of the Medicare EHR Incentive Program at Acute Care Hospitals," found surprisingly opportunistic behavior on the part of hospitals seemingly using Medicare's EHR Incentive Program to maximize the payouts they receive for Medicare patients. Their conclusions point to the need to analyze for unintended consequences when establishing programs with financial incentives. However, they caution that the potentially negative impacts suggested by this study should not be allowed to undermine the important and ongoing process of EHR adoption.

\section{CONCLUSION}

In this special issue, we have received a large number of submissions but only a limited number of selected articles can be included. As mentioned earlier, the emerging of healthcare informatics-related events has been phenomenal. The growth of research activities in healthcare informatics can be evidenced by the recent enactment of the \$50-billion Health Information Technology for Economic and Clinical Health (HITECH) ACT in the US and a similar \$120-billion act in China, as well as some other countries. We anticipate that computing-oriented healthcare informatics research will grow at a much faster pace in the coming five to ten years, which will bring together researchers in multiple disciplines to collaborate on innovative research and practical applications to solve healthcare problems.

\section{REFERENCES}

Suhair Alshehri and Rajendra K. Raj. 2013. Secure access control for health information sharing systems. In Proceedings of the IEEE International Conference on Healthcare Informatics (ICHI'13). IEEE, 277-286. 
S. Ananiadou and J. McNaught Eds. 2006. Text Mining for Biology and Biomedicine. Artech House, Boston / London.

I. Bardhan, C. Oh, and Z. Zheng. 2011. A profiling model for readmission of patients with congestive heart failure. In Proceedings of the International Conference on Information Systems (ICIS).

Xiaoxiao Dai, Meng Wu, Bradley Davidson, Mohammad Mahorr, and Jun Zhang. 2013. Image-based fall detection with human posture sequence modeling. In Proceedings of the IEEE International Conference on Healthcare Informatics (ICHI'13). IEEE, 376-380.

Joyce C. Ho, Joydeep Ghosh, and K. P. Unnikrishnan. 2013. Risk prediction of a multiple sclerosis diagnosis. In Proceedings of the IEEE International Conference on Healthcare Informatics (ICHI'13). IEEE, 551556.

T. Ryan Hoens, Marina Blanton, Aaron Steele, and Nitesh V. Chawla. 2013. Reliable medical recommendation systems with patient privacy. ACM Trans. Intell. Syst. Technol. 4, 4.

Jose M. Juarez, Jose M. Ochotorena, Manuel Campos, and Carlo Combi. 2013. Multiple temporal axes for visualising the behaviour of elders living alone. In Proceedings of the IEEE International Conference on Healthcare Informatics (ICHI'13). IEEE, 387-395.

Arif Khan and Ian McKillop. 2013. Privacy-centric access control for distributed heterogeneous medical information systems. In Proceedings of the IEEE International Conference on Healthcare Informatics (ICHI'13). IEEE, 297-286.

Ritu Khare, Jiao Li, and Zhiyong Lu. 2013. Toward creating a gold standard of drug indications from FDA drug labels. In Proceedings of the IEEE International Conference on Healthcare Informatics (ICHI'13). IEEE, 30-35.

I. Korkontzelos, T. Mu, and S. Ananiadou. 2012. ASCOT: A text mining-based web-service for efficient search and assisted creation of clinical trials. BMC Medical Informatics and Decision Making, 12(Suppl 1), S3.

Klaus Lemke. 2013. A predictive model to identify patients at risk of unplanned 30-day acute care hospital readmission. In Proceedings of the IEEE International Conference on Healthcare Informatics (ICHI'13). IEEE, 551-556.

M. Kwak, G. Leroy, J. D. Martinez, and J. Harwell. 2013. Development and evaluation of a biomedical search engine using a predicate-based vector space model. J. Biomed. Informatics 46, 5, 929-39

Y. Miyao and J. Tsujii. 2008. Feature forest models for probabilistic HPSG parsing. Computational Ling. 34 $1,35-80$.

M. Miwa, P. Thompson, and S. Ananiadou. 2012. Boosting automatic event extraction from the literature using domain adaptation and coreference resolution. Bioinformatics 28, 13, 1759-1765.

Sachin Mehta, Rajarathnam Nallusamy, Ranjeet Vinayak Marawar, and Balakrishnan Prabhakaran. 2013. A study of DWT and SVD based watermarking algorithms for patient privacy in medical images. In Proceedings of the IEEE International Conference on Healthcare Informatics (ICHI'13). IEEE, 287-276.

Yubin Park and Joydeep Ghosh. 2013. CUDIA: Probabilistic cross-level imputation using individual auxiliary information. ACM Trans. Intell. Syst. Technol. 4, 4.

Parisa Rashidi and Diane J. Cook. 2013. COM: A method for mining and monitoring human activity patterns in home-based health monitoring systems. ACM Trans. Intell. Syst. Technol. $4,4$.

T. C. Rindflesch and M. Fiszman. 2003. The interaction of domain knowledge and linguistic structure in natural language processing: Interpreting hypernymic propositions in biomedical text. J. Biomed. Informatics 36, 6, 462-77.

Y. Sasaki, Y. Tsuruoka, J. McNaught, and S. Ananiadou. 2008. How to make the most of named entity dictionaries in statistical NER. BMC Bioinformatics, 9 (Suppl 11), S5.

H. Thimblebly. 2013. Improving safety in medical devices and systems. In Proceedings of the IEEE International Conference on Healthcare Informatics (ICHI'13). IEEE.

Christopher C. Yang, Haodong Yang, Ling Jiang, and Mi Zhang, 2012. Social media mining for drug safety signal detection. In Proceedings of the ACM CIKM International Workshop on Smart Health and Wellbeing (SHB'12). ACM.

Haodong Yang and Christopher C. Yang. 2013. Harnessing social media for drug-drug interactions detection. In Proceedings of the IEEE International Conference on Healthcare Informatics (ICHI'13). IEEE, 22-29.

Shipeng Yu, Alexander van Esbroeck, Faisal Farooq, Glenn Fung, Vikram Anand, and Balaji Krishnapuram. 2013. Predicting readmission risk with institution specific prediction models. In Proceedings of the IEEE International Conference on Healthcare Informatics (ICHI'13). IEEE, 551-556.

Received February 2013; revised March 2013; accepted June 2013 